\title{
PICTORIAL REVIEW
}

\section{Initial experience with cinematic rendering for chest cardiovascular imaging}

\author{
STEVEN P ROWE, MD, PhD, PAMELA T JOHNSON, MD and ELLIOT K FISHMAN, MD
}

Department of Radiology and Radiological Science, Johns Hopkins University School of Medicine, Baltimore, MD, USA

Address correspondence to: Dr Steven P Rowe

E-mail: srowe8@jhmi.edu

\section{ABSTRACT}

Three-dimensional (3D) CT with volume rendering and maximum intensity projection is an integral component of CT interpretation for diagnosis and treatment planning of vascular pathology. Cinematic rendering is a new rendering algorithm that incorporates a more advanced lighting model than that used for volume rendering, to create photorealistic 3D CT images. The post-processing tool is not yet widely available and its ultimate clinical utility has yet to be assessed in well-designed studies. Nonetheless, the striking level of detail and enhanced depiction of 3D anatomic relationships holds potential for improvements in diagnosis, interventional or operative planning and patient-centred care. In this pictorial essay, a series of complex cardiovascular cases are presented to demonstrate the enhanced display capabilities associated with cinematic rendering, and the utility to guide patient management and understanding is discussed.

\section{INTRODUCTION}

The creation of isotropic voxel volumetric datasets from submillimeter multidetector spiralCT scanner technology made three-dimensional (3D) CT a reliable diagnostic tool to evaluate smaller vessels (cerebral, coronary, lower extremity run-off), eliminating the requirement for diagnostic arteriography in many patients. Maximum intensity projection (MIP) and volume rendering (VR) have welldefined advantages in vascular imaging and have become routine adjuncts to two-dimensional (2D) CT angiography image interpretation. A number of investigations have demonstrated the relatively higher diagnostic accuracy of volume rendering compared to MIP and equivalent or greater diagnostic accuracy compared with digital subtraction angiography, attributed to the algorithm's ability to depict 3D relationships. ${ }^{1-4}$

Cinematic rendering (CR) represents a new method of $3 \mathrm{D}$ visualization for volumetric data with the potential for even higher diagnostic accuracy owing to improvements in 3D display and anatomic detail that result from its unique lighting model. ${ }^{5,6}$ The data handling required for CR bears similarities to traditional VR in that thin-slice reconstructed CT data is stacked into a $3 \mathrm{D}$ volume, each isotropic voxel in the volume is assigned a colour and transparency based upon its component tissues as determined by known attenuation thresholds, and light is projected through the volume in order to generate the $3 \mathrm{D}$ images.
However, CR utilizes a much more complex lighting model that more accurately depicts the manner in which light acts when coming into contact with real-world objects. ${ }^{7}$ In brief, instead of the simple ray-casting method that is used for traditional VR (i.e. each ray from the pre-defined light source being used to generate the 3D image passes through voxels of varying transparency and colour within the volumetric dataset and generates a pixel in the $3 \mathrm{D}$ image that is reflective of the component tissues of each of those voxels), the global lighting model in CR incorporates information from thousands of light rays propagating through the volumetric dataset and includes effects on those light rays from scatter and from voxels adjacent to the paths of the rays. The tremendous amount of information that is conveyed through this projection method into the final 3D image allows for a photorealistic level of detail that is not possible with other $3 \mathrm{D}$ reconstruction methods.

While CR is still a nascent technique with a great deal of additional work required to define the ultimate utility of the method, for regions of complex anatomy it is likely to be of tremendous value to both interpreting imaging specialists and vascular interventionalists who are determining patient treatment plans. Additionally, as radiology strives to become a more patient-centred field of medicine, the quality of these images can facilitate explanation of disease state to patients as well as informed consent. Improvements 
in image quality are demonstrated in this case series of chest cardiovascular pathology.

\section{CLINICAL EXAMPLES}

Imaging of thoracic vasculature presents a unique set of challenges owing to the complex relationships between systemic and pulmonary arteries, in addition to artefacts that result from cardiac and respiratory motion. Retrospective and prospective gating can be used to address the motion artefact. For interpretation and display, this anatomic region is best evaluated with rendering algorithms that preserve the $3 \mathrm{D}$ relationships of these intertwined arteries. Accordingly, volume rendering and cinematic rendering are the optimal post-processing tools for pulmonary and aortic imaging.

Multidetector CT is the imaging modality of choice to evaluate acute and chronic conditions of the aorta. ${ }^{8}$ Additionally, 3D post-processing techniques have long been known to provide important information regarding the aorta. ${ }^{9}$ Although studies evaluating the advantages and disadvantages of CR relative to VR have yet to be carried out, initial clinical experience highlights the potential of CR to provide valuable information in this context. For example, endovascular repair of aortic aneurysms has become commonplace in recent years due to the minimally invasive nature of catheter-based treatment strategies in comparison to the major surgical approaches that were previously required. MIP and VR images both play a role in the pre-procedure evaluation and post-procedure follow-up of patients needing stent-graft repair of aortic aneurysms. However, the highly detailed and life-like images possible with CR may come to be a valuable asset for these complex cases that may supplant the role of other $3 \mathrm{D}$ imaging techniques, particularly traditional VR (Figures 1 and 2). Figure 1e,f also demonstrates how the parameters used to create a CR render can be changed by the imaging specialist to highlight certain aspects of a complex anatomic case. The presence of structures with varying levels of attenuation can be either added or subtracted from the 3D image and volume editing can be utilized to remove overlapping structures to allow better visualization of a finding of interest. As with any $3 \mathrm{D}$ reconstruction methodology, CR is optimally used in an interactive setting in which the windowing level, fieldof-view and perspective are manipulated by the interpreting radiologist in order to best highlight salient findings. It is expected that work-flow will be enhanced with the development of preset parameters for specific applications and anatomy.

Coarctation of the aorta is a rare condition but can produce profound flow gradients across the region of aortic narrowing leading to a low arterial flow state distally and chronically leading to the development of large collateral pathways. The appearance

Figure 1. (a) Coronal and (b) sagittal contrast-enhanced 2D CT images from a 75-year-old female patient status-postendovascular stent-graft repair of a large descending thoracic aortic aneurysm. The presence of contrast within the excluded portion of the aneurysm (white arrowhead) is consistent with an endoleak. (c) MIP image also demonstrates the endoleak (white arrowhead); MIP reconstructions can be very helpful for the identification of subtle contrast extravasation but lack information on the relative anatomic positions of overlapping vascular structures within the MIP slab. (d) VR image again shows the endoleak (white arrowhead) but adds anatomic detail that is not available in the MIP. (e, f) CR images depict the endoleak as well (white arrowheads), although with a photorealistic level of detail that is not available with traditional VR. CR, cinematic rendering; MIP, maximum intensity projection; VR, volume rendering, 2D, two-dimensional.
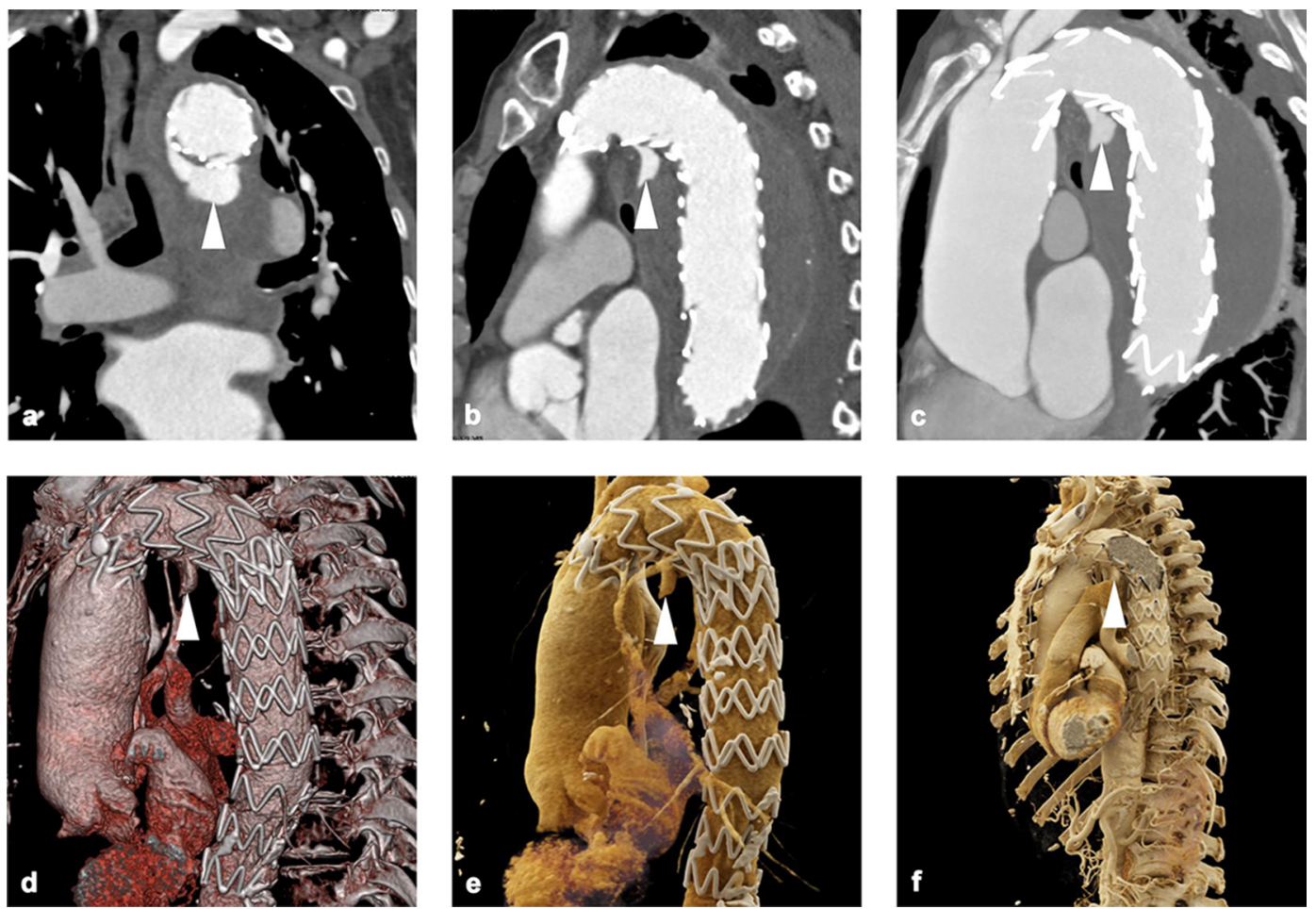
Figure 2. A 69-year-old male patient presenting with chest pain. (a) Oblique sagittal post-contrast 2D CT image demonstrating a partially thrombosed aneurysm of the descending aorta with a large penetrating ulcer (white arrowhead). (b, c) VR images from the same patient provide a 3D assessment of the ulcer (white arrowheads), although the thrombosed portion of the aneurysm is less well-appreciated. Nonetheless, the VR reconstructions provide a valuable overview and volumetric data for planning stentgraft placement. (d) CR image that again displays to good advantage the large penetrating ulcer (white arrowhead). While no specific additional information about the ulcer itself is available from the CR image relative to the VR images, the level of detail in displaying anatomic relationships to other structures [such as the left innominate artery (white arrow)] is aided by the photorealism of the image. CR, cinematic rendering; VR, volume rendering, 2D, two-dimensional, 3D, three-dimensional.
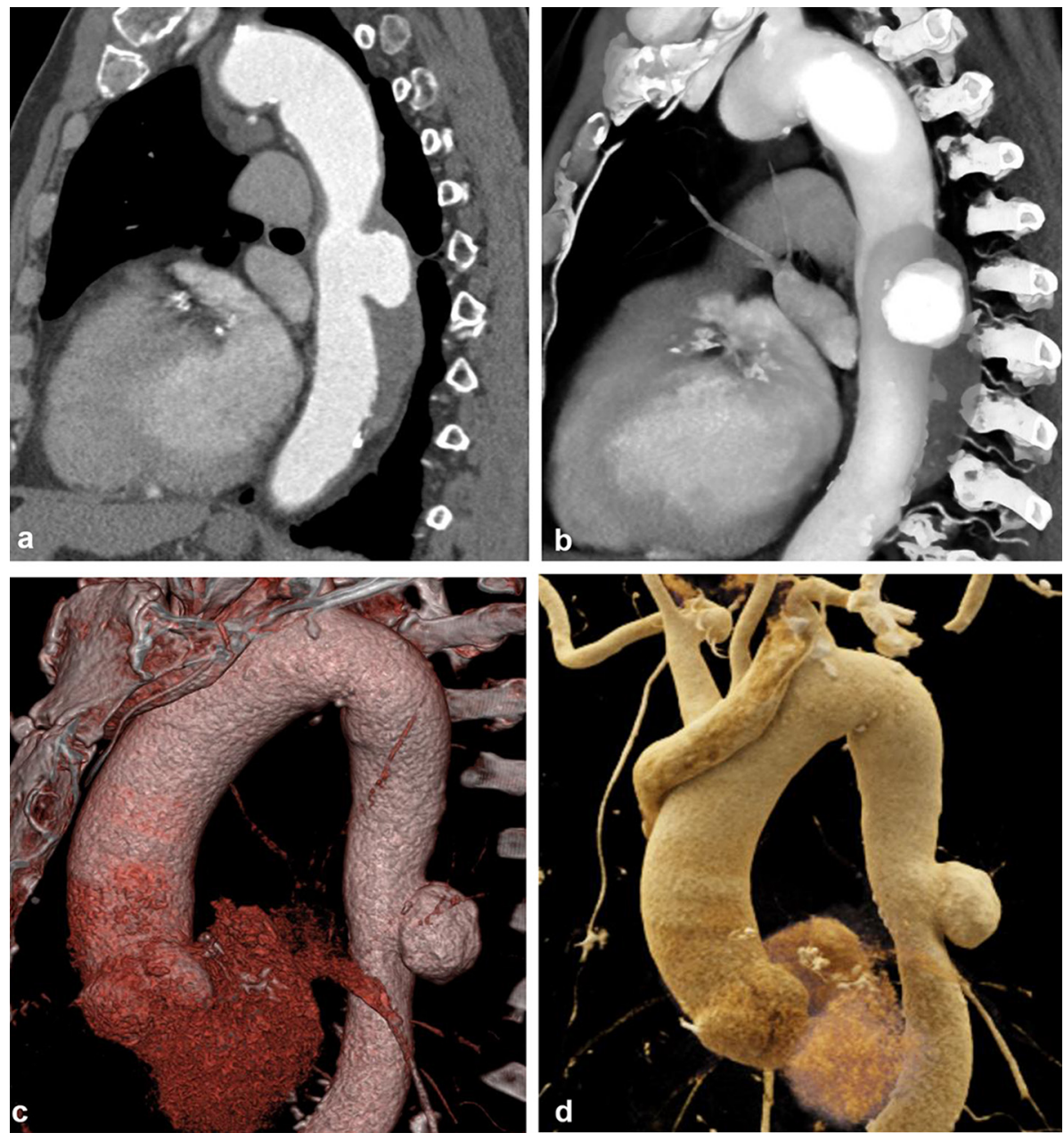

of both the primary underlying pathology of aortic narrowing and the presence of enlarged collateral vessels has been extensively described with $3 \mathrm{D}$ CT methods. ${ }^{10}$ Figure 3 provides an example of a patient with a very narrow coarctation that is depicted in high detail by CR reconstructed images. These patients also generally undergo endovascular repair. The quality of the cinematic renderings is sufficient to demonstrate this aortic pathology to patients and enhance their understanding of how the blood flow is compromised by the aortic constriction and the mechanism of repair.

Both surgical and endovascular approaches are utilized for cardiac valve replacement, and complications of artificial valves may best be evaluated using $3 \mathrm{D}$ post-processing techniques. ${ }^{11}$ Although the data are not yet available comparing prosthetic valve evaluation with $\mathrm{CR}$ to traditional VR, the highly detailed and life-like images produced through CR methodology may improve the diagnosis of subtle complications such as early bioprosthetic valve degeneration or the formation of small thrombi (Figure 4).

In next considering the pulmonary arterial vasculature, multiple important pathologic conditions merit discussion. Foremost among these is thromboembolic disease, which continues to be a significant source of morbidity and mortality. ${ }^{12}$ In the example presented in Figure 5, CR better visualizes chronic pulmonary embolism relative to traditional VR imaging, possibly as a result of intrinsically higher detail that allows for discrimination of the subtle textural difference in the vessel bearing eccentric clot that is adherent to the vessel wall. Furthermore, CR also provides highly detailed visualization of pulmonary arterial vascular conditions that may require surgical intervention such as pulmonary artery aneurysms (Figure 6) and arteriovenous malformations (Figure 7). In both Figures 6 and 7, the photorealistic shadowing effects of CR 
Figure 3. A 59-year-old male patient who was experiencing exercise intolerance and was found to have widely disparate upper and lower extremity blood pressures. (a) Axial post-contrast 2D CT image demonstrates marked narrowing/coarctation of the aorta (white arrowhead) just distal to the takeoff of the left subclavian artery. (b) CR image displays the coarctation in 3D (white arrowhead) with realistic shadowing effects and a detailed depiction of the relative positions of other vascular structures such as the immediately inferior left main pulmonary artery. (c) Another CR visualization demonstrates representative collateral vessels (bilateral internal mammary arteries, white arrows) that have arisen in this patient with a long-standing coarctation. CR, cinematic rendering; 2D, two-dimensional; 3D, three-dimensional.

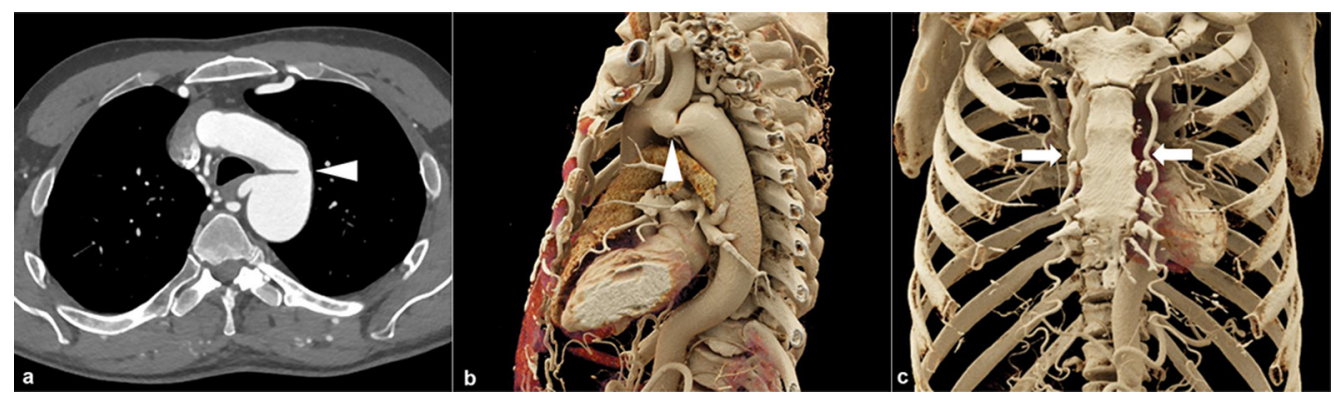

provide accurate spatial information about small vessels within the complex branching pattern of the pulmonary vasculature that are in close proximity to the pathologic structure. It is worth noting, however, that the same photorealistic shadowing can potentially obscure important structures, a potential pitfall in CR that should be understood by both the interpreting image specialist and the referring clinician?

Small vascular structures in the chest can also be evaluated with CR. MIP imaging may continue to provide the highest level of visualization very small vessels, but lacks the relative anatomic information available in both traditional VR and CR images. In our early experience, we have been able to successfully identify such pathologies as aberrant courses of coronary arteries (Figure 8) and aneurysmally dilated bronchial arteries (Figure 9). Given the need for surgical interventions in some of these small vessel pathologies, and the ability of CR to produce photorealistic renderings that display both the vessels and adjacent anatomy, we look forward to the future studies that will assess the utility of CR in this context.

Figure 4. A 92-year-old male patient with history of aortic stenosis initially treated with an open surgical procedure to place a bioprosthetic aortic valve and subsequently revised by endovascular replacement of a CoreValve. (a, c) VR images demonstrating the CoreValve (white arrowheads) inside the bioprosthetic valve (white arrows). The patient also has a patent saphenous veinto-left anterior descending artery bypass graft [thin white arrow in (c)]. (d-f) CR images also demonstrate the CoreValve [black arrowhead in (d) and white arrowheads in (e, f)] within the older bioprosthetic valve [black arrow in (d) and white arrow in (e)]. The patient's patent saphenous vein graft is also apparent in (d) (thin black arrow). The photorealistic quality of the VR images may find eventual application in aiding vascular surgeons and other interventionalists by allowing the diagnosis of subtle thromboses or other valve-related pathologies that are not as apparent with less detailed visualizations.

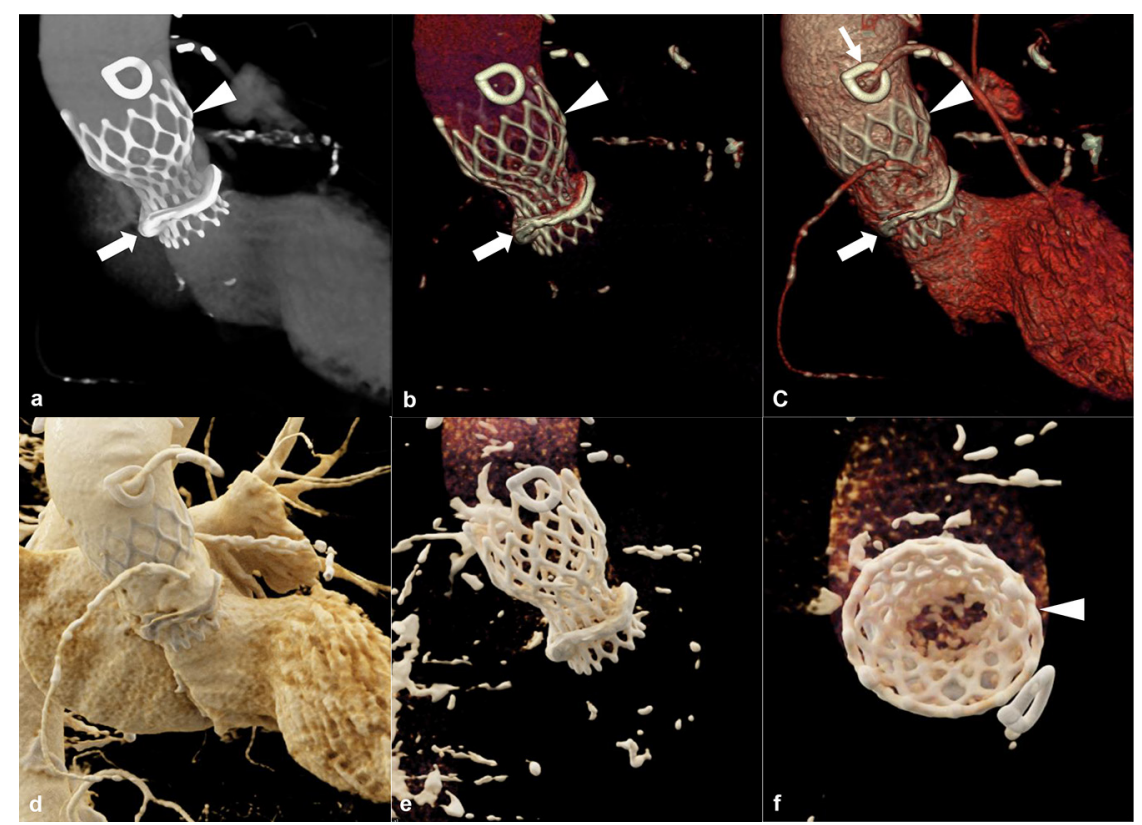


Figure 5. A 65-year-old male patient with a history of chronic thromboembolic disease. (a, b) Axial post-contrast 2D CT images demonstrating eccentric filling defects in dilated left lower lobe pulmonary arterial branches (white arrowheads) compatible with chronic pulmonary embolism. (c, d) VR images demonstrate the dilated lower lobe pulmonary arteries (white arrowheads), although the eccentric filling defects from the clots are not well seen on these images. (e, f)CR images [(f) is a blown up detail from (e)] again show the dilated lower lobe pulmonary arteries (white arrowheads), but due to the higher detail of CR, there is a subtle textural change in the left lower lobar pulmonary artery [best seen in (f)] that is consistent with the patient's chronic pulmonary embolism.

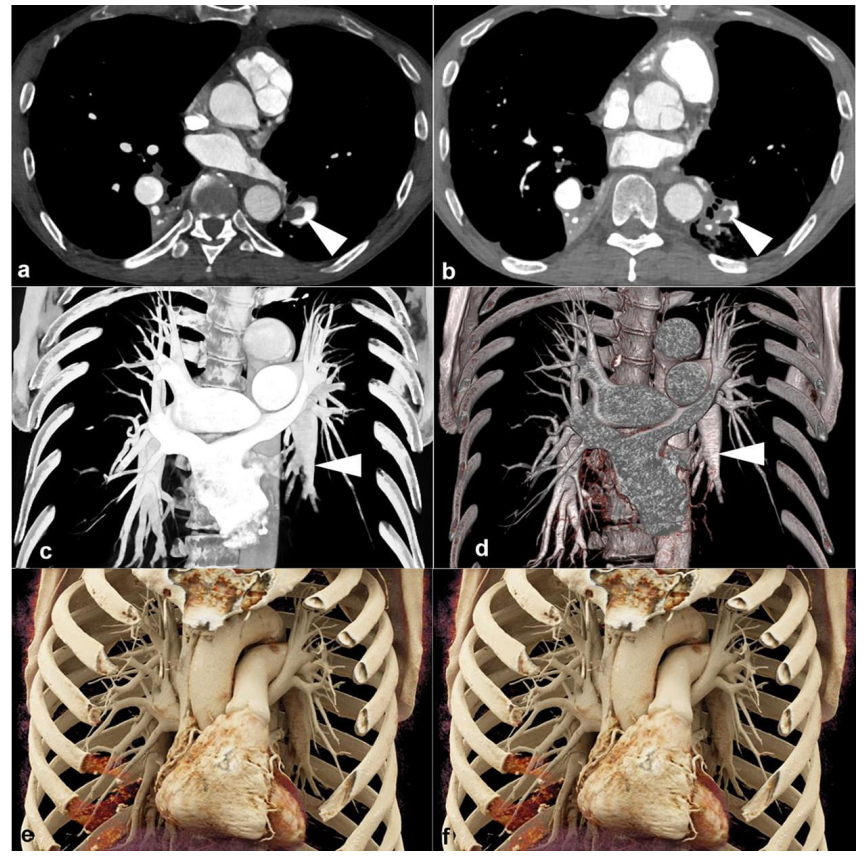

Figure 6. A 52-year-old female patient with history of pulmonary artery aneurysm of unknown aetiology. (a) Axial post-contrast 2D CT image demonstrating marked enlargement of the main pulmonary artery (measuring $5.2 \mathrm{~cm}$, white arrowheads). (b) VR image also demonstrates the dilated main pulmonary artery (white arrowheads), with improved visualization of the relationship of the aneurysmal vessel relative to other vascular structures such as the aortic arch and the branching pulmonary vessels. (c) CR visualization once more shows the aneurysmal main pulmonary artery (white arrowheads) and its 3D relationship to other major vessels. The photorealistic detail and shadowing available with $C R$ creates high contrast that better highlights the smaller pulmonary vessels that overlie the main pulmonary artery in this view (white arrow). CR, cinematic rendering; VR, volume rendering; 2D, two-dimensional; 3D, three-dimensional.

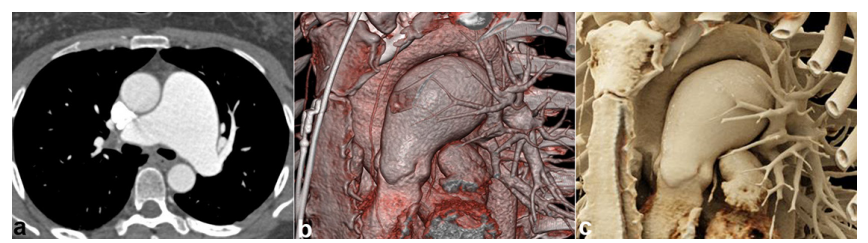

Figure 7. Images from a 70-year-old female patient with hereditary haemorrhagic telangiectasia. (a) Sagittal post-contrast 2D CT image demonstrates a large left lower lobe arteriovenous malformation (white arrowhead) that has been treated with an Amplatzer occlusion device. There is no evidence of contrast flow within the malformation. (b) MIP image again demonstrates the arteriovenous malformation (white arrowhead), although relative to the $2 \mathrm{D}$ image it is much more apparent that a number of small pulmonary arterial branches wrap around the malformation. (c) CR image displays the arteriovenous malformation with photorealistic detail despite its lack of contrast enhancement, and also conveys the relationship of the malformation to the nearby pulmonary arterial branches. Given the method's derivation from traditional VR, $\mathrm{CR}$ maintains the relative anatomic relationships of structures in a superior manner to MIP. Had this patient failed endovascular therapy and required a surgical approach for treatment, the accurate visualization of small adjacent vessels would have been of particular importance.

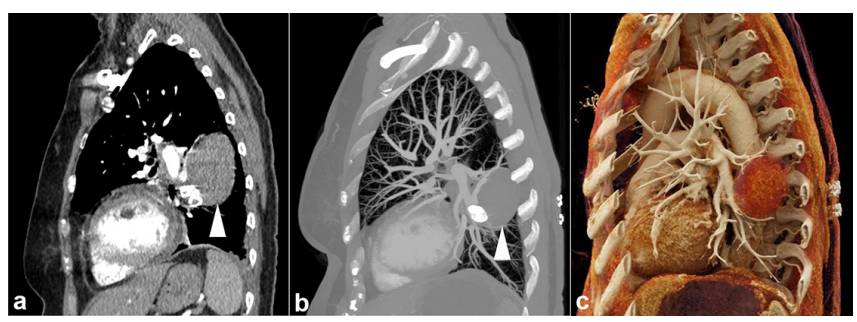

Tumours in close association with major vascular structures also require $3 \mathrm{D}$ reconstructions for pre-operative planning. CR provides valuable information to the surgeons undertaking the complex task of resecting masses that have insinuated themselves among major vascular structures. Although the patient in

Figure 8. A 59-year-old male patient undergoing a work-up for chest pain. (a) MIP reconstruction demonstrating that the patient has an anomalous course of the left circumflex artery which arises from the right coronary artery (white arrowhead). (b) VR image again shows the left circumflex artery arising from the right coronary artery (white arrowhead). With its improved ability to represent relative anatomic relationships in comparison to MIP, this image makes clear that the anomalous left circumflex artery takes a posterior course behind the aorta (a benign coronary artery anomaly). (c) The more realistic portrayal of the anomalous left circumflex artery (white arrowhead) that is afforded by CR visualization hints at the potential utility of this 3D methodology to assist in planning complex cardiovascular surgeries in patients that may have malignant anomalous coronary vessels. CR, cinematic rendering; MIP, maximum intensity projection; $\mathrm{VR}$, volume rendering; 3D, three-dimensional.

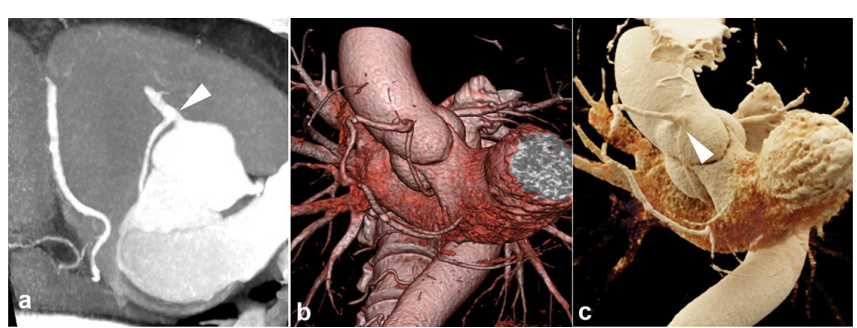


Figure 9. Images from an 87-year-old female patient who was presenting for follow-up of mild aneurysmal dilation of the ascending aorta. (a) Axial post-contrast 2D CT image demonstrates dilated vascular structures in the mediastinum (white arrowhead). (b, c) VR images demonstrate another of these dilated vascular structures (white arrowheads), which in 3D are more apparently aneurysms of the bronchial arteries. (d-f) CR images again demonstrating the multiple bronchial artery aneurysms (white arrowheads depict the aneurysm that arises at the origin of one of the bronchial arteries from the descending aorta). This patient was not felt to be a candidate for any therapy of these lesions given significant comorbidities and no cause for the bronchial artery aneurysms was ever uncovered. Nonetheless, the realistic shadowing provided by $\mathrm{CR}$ and that is most apparent in ( $f$ ) provides very clear representation of the relationships between the aneurysms and other structures including the heart and descending aorta and would be of value in surgical planning. $C R$, cinematic rendering; VR, volume rendering; 2D, two-dimensional; 3D, threedimensional.

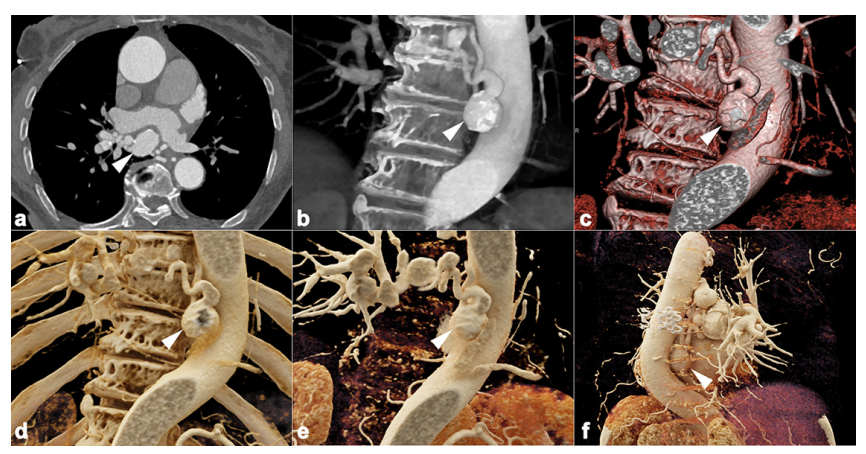

Figure 10 was not a candidate to undergo resection of his large mediastinal conglomerate lymph node mass, the ability of CR to display the relationship between the mass and the great vessels of the mediastinum provides a great deal of promise that this technique will manifest itself in clinically useful ways.

\section{CONCLUSIONS}

The examples provided in this pictorial essay demonstrate the photorealistic depictions of chest cardiovascular pathologies afforded by CR. Given the need to represent such pathologies in arealistic manner as possible in order to assist in making the most appropriate patient management decisions and in directly communicating information to patients, it is quite likely that such renderings will play an important role in the future as they
Figure 10. A 66-year-old male patient with known diagnosis of pulmonary large cell neuroendocrine tumour. (a) Axial post-contrast 2D CT image demonstrating a confluent lymph node mass (white arrowhead) occupying much of the superior mediastinum with encasement of the trachea and interdigitation among multiple vascular structures. (b) VR image also demonstrates the mass (white arrowhead) which abuts and/or narrows the superior vena cava, the innominate veins, the aorta and major arterial branches arising from the aortic arch, and the main, left and right pulmonary arteries. (c, d) $C R$ visualizations; the photorealism of these renders allows for the depiction of tumour infiltrating between the left and right pulmonary arteries [white arrow in (c)] and between the ascending aorta and left pulmonary artery [black arrowhead in (d)]. While this patient was not a surgical candidate, the added detail from $C R$ relative to other 3D techniques may prove quite valuable in surgical planning in regions of complex vascular anatomy. $\mathrm{CR}$, cinematic rendering; $\mathrm{VR}$, volume rendering; 2D, two-dimensional; 3D, three-dimensional.

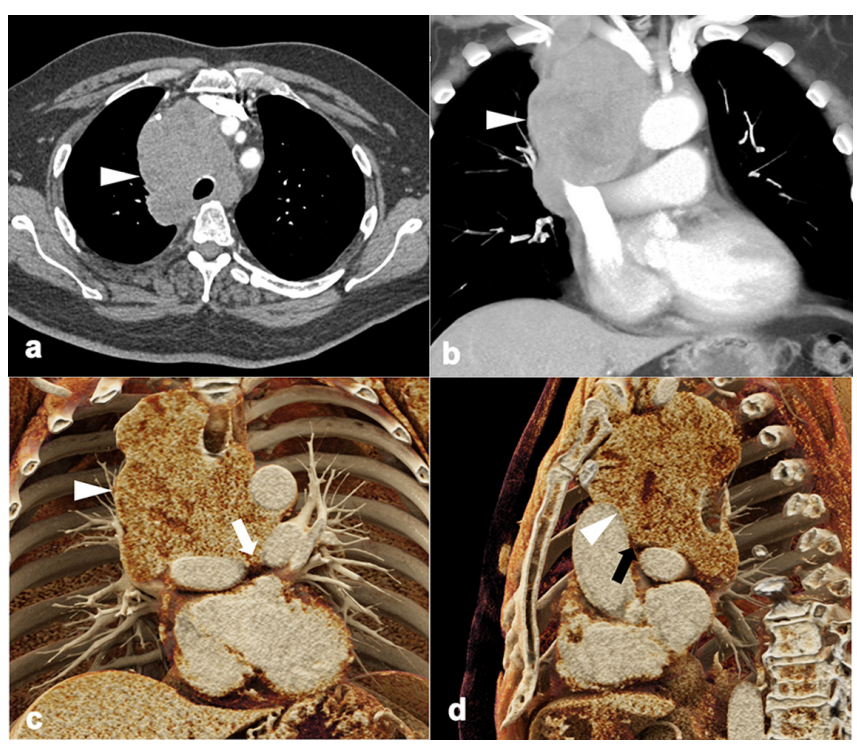

become more widely clinically available. Furthermore, given the expanding role of $3 \mathrm{D}$ printing in treatment planning for cardiovascular surgery, ${ }^{13}$ these hyper-realistic renderings are likely to be of tremendous value to surgeons and interventionalists given their ability to recapitulate the visual experience of the 3D printed models. Well-thought-out studies that establish the advantages and disadvantages of this new technique are needed and are forthcoming.

\section{REFERENCES}

1. Johnson PT, Halpern EJ, Kuszyk BS, Heath DG, Wechsler RJ, Nazarian LN, et al. Renal artery stenosis: CT angiography-comparison of real-time volume-rendering and maximum intensity projection algorithms. Radiology 1999; 211: 337-43. doi: https://doi.org/10. 1148/radiology.211.2.r99ap17337
2. Addis KA, Hopper KD, Iyriboz TA, Liu Y, Wise SW, Kasales CJ, et al. CT angiography: in vitro comparison of five reconstruction methods. AJR Am J Roentgenol 2001; 177: 1171-6. doi: https://doi.org/10.2214/ajr.177. 5.1771171
3. Villablanca JP, Martin N, Jahan R, Gobin YP, Frazee, Duckwiler G, et al. Volumerendered helical computerized tomography angiography in the detection and characterization of intracranial aneurysms. J Neurosurg 2000; 93: 254-64. doi: https:// doi.org/10.3171/jns.2000.93.2.0254 
4. Villablanca JP, Hooshi P, Martin N, Jahan R, Duckwiler G, Lim S, et al. Three-dimensional helical computerized tomography angiography in the diagnosis, characterization, and management of middle cerebral artery aneurysms: comparison with conventional angiography and intraoperative findings. J Neurosurg 2002; 97: 1322-32. doi: https://doi.org/10. 3171/jns.2002.97.6.1322

5. Dappa E, Higashigaito K, Fornaro J, Leschka S, Wildermuth S, Alkadhi H. Cinematic rendering - an alternative to volume rendering for $3 \mathrm{D}$ computed tomography imaging. Insights Imaging 2016; 7: 849-56. doi: https://doi.org/10.1007/ s13244-016-0518-1

6. Eid M, De Cecco CN, Nance JW, Caruso D, Albrecht MH, Spandorfer AJ, et al. Cinematic rendering in CT: a novel, lifelike 3D visualization technique. AJR Am J Roentgenol 2017; 209: 370-109. doi: https://doi.org/10. 2214/AJR.17.17850
7. Johnson PT, Schneider R, LugoFagundo C, Johnson MB, Fishman EK. MDCT angiography with $3 \mathrm{D}$ rendering: a novel cinematic rendering algorithm for enhanced anatomic detail. AJR Am J Roentgenol 2017; 209: 309-412. doi: https:// doi.org/10.2214/AJR.17.17903

8. Meyersohn NM, Ghemigian K, Shapiro MD, Shah SV, Ghoshhajra BB, Ferencik M. Role of computed tomography in assessment of the thoracic aorta. Curr Treat Options Cardiovasc Med 2015; 17: 395. doi: https://doi.org/10. 1007/s11936-015-0395-9

9. Bartolozzi C, Neri E, Caramella D. CT in vascular pathologies. Eur Radiol 1998; 8: 679-84. doi: https://doi.org/10.1007/ s003300050459

10. Becker C, Soppa C, Fink U, Haubner M, Müller-Lisse U, Englmeier KH, et al. Spiral $\mathrm{CT}$ angiography and $3 \mathrm{D}$ reconstruction in patients with aortic coarctation. Eur Radiol 1997; 7: 1473-7. doi: https://doi.org/10.1007/ s003300050319
11. Ruggieri VG, Haigron P, Wang Q Esneault S, Madeleine R, Heautot JF, et al. CT-scan images preprocessing and segmentation to improve bioprosthesis leaflets morphological analysis. Med Hypotheses 2013; 81: 86-93. doi: https://doi. org/10.1016/j.mehy.2013.03.032

12. Raja AS, Greenberg JO, Qaseem A, Denberg TD, Fitterman N, Schuur JD.Evaluation of patients with suspected acute pulmonary embolism: best practice advice from the xlinical guidelines committee of the American college of physicians. Ann Intern Med 2015; 163: 701-11. doi: https://doi.org/10.7326/M141772

13. Bartel T, Rivard A, Jimenez A, Mestres CA, Müller S. Medical threedimensional printing opens up new opportunities in cardiology and cardiac surgery. Eur Heart J 2017. Epub ahead of print. doi: https://doi.org/10.1093/eurheartj/ ehx016 